\title{
BMJ Open Intensive rehabilitation programme for patients with subacute stroke in an inpatient rehabilitation facility: describing a protocol of a prospective cohort study
}

\author{
Monique Messaggi Sartor (10 , 1,2 Jennifer Grau-Sánchez,, ${ }^{3,4}$ Anna Guillén-Solà, 1,2,5 \\ Roser Boza, ${ }^{1}$ Josep Puig, ${ }^{6}$ Cathy Stinear, ${ }^{7}$ Andrea Morgado-Perez, ${ }^{1,2}$ \\ Esther Duarte ${ }^{1,2,5}$
}

To cite: Sartor MM, GrauSánchez J, Guillén-Solà A, et al. Intensive rehabilitation programme for patients with subacute stroke in an inpatient rehabilitation facility: describing a protocol of a prospective cohort study. BMJ Open 2021;11:e046346. doi:10.1136/ bmjopen-2020-046346

- Prepublication history and additional supplemental material for this paper are available online. To view these files, please visit the journal online (http://dx.doi.org/10.1136/ bmjopen-2020-046346).

Received 27 0ctober 2020 Accepted 29 July 2021

Check for updates

(c) Author(s) (or their employer(s)) 2021. Re-use permitted under CC BY-NC. No commercial re-use. See rights and permissions. Published by BMJ.

For numbered affiliations see end of article.

Correspondence to Dr Monique Messaggi Sartor; mosartor@gmail.com

\section{ABSTRACT}

Introduction Rehabilitation is recognised as a cornerstone of multidisciplinary stroke care. Intensity of therapy is related to functional recovery although there is high variability on the amount of time and techniques applied in therapy sessions. There is a need to better describe stroke rehabilitation protocols to develop a better understanding of current practice increasing the internal validity and generalisation of clinical trial results. The aim of this study is to describe an intensive rehabilitation programme for patients with stroke in an inpatient rehabilitation facility, measuring the amount and type of therapies (physical, occupational and speech therapy) provided and reporting functional outcomes.

Methods and analysis This will be a prospective observational cohort study of patients with subacute stroke admitted to our inpatient rehabilitation facility during 2 years. A therapy recording tool was developed in order to describe the rehabilitation interventions performed in our unit. This tool was designed using the Delphi method, literature search and collaboration with senior clinicians. Therapists will record the time spent on different activities available in our unit during specific therapy sessions. Afterwards, the total time spent in each activity, and the total rehabilitation time for all activities, will be averaged for all patients. Outcome variables were divided into three different domains: body structure and function outcomes, activity outcomes and participation outcomes and will be assessed at baseline (admission at the rehabilitation unit), at discharge from the rehabilitation unit and at 3 and 6 months after stroke.

Ethics and dissemination This study was approved by the Medical Research Committee at Hospital del Mar Research Institute (Project ID: 34/C/2017). The results of this study will be presented at national and international congress and submitted for publication in peer-reviewed journals.

Trial registration number NCT04191109.

\section{INTRODUCTION}

Stroke is the second cause of death and disability worldwide. ${ }^{12}$ Improvements in acute management have reduced stroke mortality
Strengths and limitations of this study

- This study will provide a description of an intensive rehabilitation programme for patients with subacute stroke delivered at our inpatient rehabilitation unit.

- Another crucial contribution of this study is that the clinical assessments of the patients are not limited to the inpatient period, but also extend to the longterm phase.

- One of the strengths of this study is that treatment registry data has been agreed between a multidisciplinary team of the rehabilitation unit, reflecting actual clinical practice.

- The data collected in this study will rely on the information provided by the therapists, and although all participating therapists have agreed to provide reliable data when reporting therapy sessions, there might be a social desirability bias.

- This study does not consider the assessments of patient-centred aspects, which may limit the understanding of patient's experience.

rates in developed countries; however, the burden of stroke on individuals, caregivers and societies increases. ${ }^{3}$ Many stroke survivors experience motor, sensory, perceptual and cognitive deficits, needing rehabilitation in the following months after the stroke. ${ }^{4}$ Stroke rehabilitation aims to promote functional recovery and autonomy through restitution, substitution and compensation of functions to achieve the highest possible level of functional recovery. ${ }^{5}$ Rehabilitation is a patient-centred process delivered by a multidisciplinary team, including medical doctors, physical, occupational and speech therapists, nurses, social workers and neuropsychologists. ${ }^{6}$ After discharge from the stroke unit, the post-acute inpatient care services for 
patients with stroke include rehabilitation facilities and long-term care hospitals. ${ }^{6}$ Inpatient rehabilitation facilities provide hospital-level care and should offer intensive programmes of therapy. ${ }^{7}$ Patients treated in these inpatient rehabilitation facilities have better functional outcomes and higher return rates to community living than those treated in general wards or long-term care hospitals. ${ }^{6-11}$ Factors such as age, the need of 24-hour medical care, previous functional independence, cognitive deficits and severity are taken into account to admit patients in inpatient rehabilitation facilities. However, hospital bed availability and health insurance coverage determine access to inpatient rehabilitation facilities. ${ }^{11}$ In most European countries, stroke rehabilitation services are not homogeneously distributed in the territory, existing a wide range of rehabilitation centres with different admission criteria ${ }^{12}$ that vary across countries and regions within the same country. Therefore, it is crucial to continue investigating inpatient rehabilitation facilities' benefits and claim for the financial resources needed to ensure the most optimal and effective care.

In the following weeks after the stroke, there is a time-limited period of increased plasticity that favours the establishment of new connections and forms of experience-dependent plasticity. ${ }^{13}$ One of the biggest challenges in rehabilitation is to understand how to modulate the mechanisms of increased plasticity through the different elements of the rehabilitation process. In this vein, the intensity and the type of therapeutical activities are central elements that influence the degree of functional recovery. ${ }^{14}$

The relationship between the amount of therapy and recovery has been extensively documented in animal models and in some clinical studies. ${ }^{814-16}$ Intensive rehabilitation facilities usually provide rehabilitation programmes that comprise 3 hours of therapy per day following international standards. ${ }^{6}$ Training intensity is a relevant component of learning, but 3 hours of therapy do not guarantee 3 hours of training. Time is lost in transportation within the facility, preparation of activities and tasks and other activities that are not directly related to practice. ${ }^{15} 17$ Different studies measuring the amount of practice in stroke rehabilitation describe that the training dose provided differs substantially from what it is prescribed. ${ }^{18}$ There is a need to investigate current practice models in inpatient rehabilitation facilities and describe how intensive rehabilitation programmes are implemented. ${ }^{1920}$

In the literature, therapeutical plans are usually reported by defining their aims, but activities and tasks during therapy sessions are often weakly described. Interventions and the content of sessions are highly variable between studies, limiting the generalisation of results and contributing to what is known as the 'black box' of rehabilitation. ${ }^{21}$ For this reason, there is a need to better describe stroke rehabilitation protocols to develop a better understanding of the current practice, the internal validity and generalisation of clinical trial results. ${ }^{22}$ Few studies have examined therapy content and defined which components or approaches are most effective for achieving the highest level of functional recovery. ${ }^{23-28}$ Bode $e t a l^{25}$ grouped activities within the rehabilitation programme into the following categories: evaluation, screening, function-focused activities, impairment focus activities, discharge planning and case management. In this study, therapists recorded the number of 15-min units spent primarily in each activity throughout the hospital stay. Lang $e t a l^{26}$ registered the number of activity repetitions during physical and occupational therapy sessions. The Stroke Physiotherapy Intervention Recording tool (SPIRIT) is a recording system developed by Tyson and Selley $^{27}$ that includes all the interventions physiotherapists use to treat postural control post stroke. Activities were divided into six categories: upper extremity movements, lower extremity movements, gait, stair climbing, transfers and balance activities. Veerbeek et $a l^{28}$ tested the effectiveness of different physiotherapy interventions classified into seven domains based on a consensus between authors. This classification comprised gait and mobility, arm-hand activities, activities of daily living, physical fitness, other interventions, the intensity of practice and neurological treatment approaches. Aside from these studies, there is a shortage of registries with prospective uniform, repeated and time-fixed measurements of determinants and measures of functional outcomes after stroke and none have been carried out in Spain (PSROP, CERISE, UDS) ${ }^{29-31}$ In Catalonia, the Stroke Programme created a stroke population-based registry (SONIIA) with external monitoring of data completeness assessing the quality of reperfusion therapies delivered to patients with ischaemic stroke since 2011. However, this database does not yet include specific information regarding the rehabilitation process and long-term functional outcomes.

The study aims to describe an intensive rehabilitation programme for patients with stroke at our inpatient rehabilitation facility, measuring the amount and type of therapies (physical, occupational and speech therapy) and reporting functional outcomes. Based on previous research, we hypothesise that the amount of therapy delivered is less than what it is planned, and that most therapy activities will be directed to reduce deficits in body functions. In this vein, we also expect that patients show major improvements on body functions during their stay at our inpatient rehabilitation facility, and that recovery of autonomy in activities of daily living will be more prominent at 3-months and 6-months post discharge.

\section{METHODS AND ANALYSIS \\ Design}

This will be a prospective observational cohort study of patients with subacute stroke admitted to our inpatient rehabilitation facility during 2 years. This protocol will be described following the Strengthening the Reporting of Observational Studies in Epidemiology guidelines. ${ }^{32}$ This cohort will be part of the BRAIN-CONNECTS study, 
which is a multicentre prospective study to determine the value of brain connectivity analysis in predicting functional outcomes in the rehabilitation of patients with subacute stroke. The BRAIN-CONNECTS: Brain Connectivity during Stroke Recovery and Rehabilitation study is approved by the Ethics Committee of the Hospital del Mar Medical Research Institute (Barcelona, Spain; Project ID: 34/C/2017).

\section{Study setting}

This study will be conducted at the Physical Medicine and Rehabilitation Department of Hospitals del Mar i l'Esperança, a tertiary referral hospital in the city of Barcelona (Catalonia, Spain). This centre offers intensive inpatient rehabilitation programmes for patients with stroke who have a good functional prognosis according to the following criteria: (i) no major cognitive deficits affecting comprehension (Montreal Cognitive Assessment (MoCA) $\geq 20$ ), (ii) low comorbidity (Charlson Index $<3$ ) and (iii) functional independence before the stroke (Modified Rankin Scale (mRS) $\leq 2$ ).

In Catalonia, after the acute care patients with stroke may be discharged to: (a) hospital with intensive inpatient rehabilitation programmes, (b) social-health centres (convalescence or long-stay centres) or (c) home with community rehabilitation (outpatient, daycare or home care). Catalonia has a population of 7.5 million and an organised and highly territorialised stroke care system administered by the Stroke Programme, an organisation created in 2004 by the Catalan Health Department. ${ }^{33}$ The code stroke protocol covers all the territory and has been a key element to ensure the fast transfer to hospitals with stroke units, and the availability of intravenous thrombolysis or endovascular therapy. Besides the transfer network, a rehabilitation advisory group within the Stroke Programme agreed to a set of inclusion criteria for each rehabilitation setting and established a territorial organisation to access intensive rehabilitation services. The Neurological Rehabilitation Unit, part of the Physical Medicine and Rehabilitation Department of Hospitals del Mar i l'Esperança, was accredited as a reference centre in Barcelona area for intensive inpatient rehabilitation programmes admitting patients with stroke from three tertiary hospitals (Hospital del Mar, Hospital Clínic and Hospital de Sant Pau i la Santa Creu). This unit admits yearly around 150 acute and early patients with subacute stroke (average time from stroke onset in 2018: 8.7 days), who receive intensive inpatient rehabilitation ( $\geq 3$ hours daily) with early supported discharge (average length of stay in 2018: 16.37 days). The Neurological Rehabilitation Unit has 18 beds, three medical doctors specialised in physical medicine and rehabilitation, four physiotherapists, two occupational therapists, one speech therapist, one social worker and one neuropsychologist. A team of nurses and nurse assistants trained in neurological rehabilitation take care of patients during the hospital stay (ie, medication, early mobilisation, sphincter control and dysphagia).

\section{Participants}

Patients with subacute stroke involved in an inpatient intensive rehabilitation programme at the Department of Physical Medicine and Rehabilitation at the Hospitals del Mar $i$ de l'Esperança will be screened for recruitment. Eligibility criteria will be: (1) aged 18 or over, (2) first-ever intracerebral ischaemic or haemorrhagic stroke confirmed by neuroimaging, (3) less than 3 weeks since stroke onset, (4) moderate-to-severe impairment (National Institute of Health Stroke Scale(NIHSS) score between 4 and 13), (5) functional independence before the stroke (Rankin $\leq 2)$, (6) no major cognitive deficits affecting comprehension (MoCA $\geq 20$ ) and (7) ability to understand Spanish and/or Catalan. Patients with any other neurological or psychiatric condition will be excluded.

The recruitment of patients will be performed by the medical doctors of the unit, who will provide oral and written information to patients and caregivers. Patients who agree to participate will sign an informed consent form. The sample size is calculated with the GRAMO programme,${ }^{34}$ using Stroke Impact Scale mobility domain as the main variable of interest. Accepting an alpha risk of 0.05 and a beta risk lower than 0.2 in a bilateral contrast, 63 participants are required to detect an increase over time of at least 4.5 points, assuming a SD of 12 points and a $10 \%$ loss to follow-up.

\section{Patient and public involvement}

Patients or the public were not involved in the design, conduct, reporting or dissemination plans of our research.

\section{Intensive inpatient rehabilitation programme}

The intensive inpatient rehabilitation programme for patients with subacute stroke aims at regaining lost body functions and recovering autonomy in basic activities of daily life. The rehabilitation programme is a patientcentred process with cyclic stages that include (i) evaluation of the patient's need, (ii) collaborative goal setting, (iii) therapeutical interventions and (iv) re-evaluation. ${ }^{35}$ Each patient has an assigned medical doctor, and a physical, occupational and speech therapist. At the patient's arrival to the Neurological Rehabilitation Unit, the patient meets with the medical doctor, who performs a global neurological and functional evaluation, provides general information about the stay at the unit and discusses short-term goals with the patient and family. A non-instrumental swallowing assessment is performed to screen for signs and symptoms of dysphagia, which, if positive, is completed with a videofluoroscopic swallowing study. Physical, occupational and speech therapists interview the patient and perform specific evaluations regarding the patient's mobility, performance in activities of daily life and communication, respectively. Within the first days of the patient's stay, a neuropsychologist performs a comprehensive evaluation focused on examining orientation, attention, executive functions, visuospatial function, memory and language deficits. The results of all these evaluations are used to set specific 
goals with the patient. The team meets weekly to review the patient's progress and redefine specific goals, and uses clinical management software to share information about each patient.

The intervention consists of at least three scheduled hours of therapy (physical, occupational and speech therapy) per day, 6 days per week. Physiotherapy sessions address global mobility, balance, transfers and walking. During occupational therapy sessions, body functions such as movement, sensation, perception and cognition are trained as well as activities of daily living. Speech therapy sessions are focused on dysphagia management, enhancing language skills and recovering from motor speech disorders. Sessions are individual, tailored to the patient's needs and graded by difficulty.

Occupational therapy sessions are sometimes performed in the patient's room, especially when training dressing, eating, toilet use, personal hygiene and transfers. Otherwise, sessions are carried out in the physical, occupational or speech therapy department, where, although working individually, patients are together and can share their difficulties and progress with each other, promoting peer support. Therapy sessions are spread throughout the day according to the patient's tolerance and needs and special attention is paid to provide sufficient rest periods during the day. Patients are allowed to receive visits from family members and friends.

During the stay, patients and caregivers are invited to attend to a 1-hour educational session led by an occupational therapist. This session aims to provide information, training and support on how to manage stroke deficits and consequences. This information and training intervention has been shown to have a positive impact on patients' and caregivers' satisfaction and perceived support. ${ }^{36}$

Since every patient has an assigned therapist for each discipline, this allows the establishment of therapeutical relationships and patients can express their concerns and feelings during their hospital stay. Importantly, all members of the team are trained to provide emotional support to the patient and caregivers.

The medical doctor together with the social worker coordinates the immediate post-discharge care at home or in the community. Discharge is planned with the patient and caregiver and interviews are performed to gather information about the home environment and social support. Most of the patients continue receiving outpatient rehabilitation at the hospital, which starts the following day after discharge. Other patients might receive outpatient rehabilitation at different centres or at home. In these cases, the social worker coordinates with the receiving teams or community-based agencies and the medical doctor prepares a medical report to ensure continuity in rehabilitation objectives and treatments.

\section{Register of the amount of therapy delivered}

The register of activities delivered in each scheduled therapy (physiotherapy, speech and occupational therapy) was developed on the basis of the proposals of Bode $e t a l^{25}$ and Veerbeek $e t a l .{ }^{28}$ An advisory group formed by one medical doctor, two physiotherapists, one occupational therapist and one speech therapist proposed a first set of therapy interventions. Only interventions and activities available in our rehabilitation programme were included, together with those that the group considered that should be added since they are part of our daily practice. Finally, the list was revised to reach enough consensus between the group. This register allows a daily recording of the number of minutes performed in each activity stratified by discipline during specific treatment sessions and thus know what interventions are carried out and how much time is dedicated to each of them (supplementary file: online supplemental file 1: registry tool OT, online supplemental file 2: registry tool SL therapy and online supplemental file 3: registry tool PT). The therapist responsible for the patient's treatment will be instructed to fill in the register, writing down the amount of time devoted to each activity during treatment session. At the end of each session, the total time spent in each activity and the total time for all activities will be calculated. The total time spent in each activity and the total rehabilitation time for all activities will be averaged for all patients.

\section{Treatment compliance and safety}

Compliance will be assessed according to (i) attendance rate (the number of planned sessions vs the number of sessions attended) and (ii) reasons for training interruption (two or more days without training session) such as fatigue, dizziness or medical instability and training session modification (dose reduction or early termination of the individual session). Safety will be assessed by recording any adverse event related to the rehabilitation programme.

\section{Evaluation of patients}

\section{Baseline variables}

Clinical and demographic variables will be collected at baseline (up to 48 hours after the admission at the neurorehabilitation unit). These include age, sex, body mass index, level of education, social status and handedness. Clinical history related to risk factors for stroke, NIHSS score at discharge from the stroke unit, stroke location using Oxford classification and stroke aetiology following the TOAST classification. Reperfusion treatment, Charlson Index and sphincter control will be collected from medical records. Data about the premorbid functioning of the patient will be collected at admission on the intensive rehabilitation facility by using the Barthel Index, which measures the individual's performance in activities of daily living, ${ }^{37} 38$ and the Functional Ambulation Category that is a clinical gait assessment scale, which distinguishes six levels of walking ability based on the amount of physical therapy required. ${ }^{39}$ 


\section{Outcomes}

According to the International Classification of Functioning, Disability and Health (ICF, WHO, 2001), the outcome variables are divided into three domains: body structure and function, activity level and participation measurements. Outcomes will be collected at baseline (within 48 hours of admission to the neurorehabilitation unit), at discharge from the unit and at 3 and 6 months post stroke.

\section{Body structure and function outcomes}

The Fugl-Meyer Assessment of motor recovery after stroke will be used to evaluate upper and lower limb motor impairment. Performance is rated on a 3-point ordinal scale from 0 to 2 , with a maximum score of 66 , higher scores indicate minimal or no impairment. ${ }^{40}$ The MoCA will be used to assess the global cognitive status of patients. The MoCA is a brief cognitive screening with high sensitivity and specificity for detecting mild cognitive impairment. ${ }^{41}$ The Apathy Evaluation Scale will be used to address characteristics of goal-directed behaviour that reflect apathy including behavioural, cognitive and emotional indicators. This tool quantifies and characterises apathy in adult patients using 18 specific items and the score ranges from 18 to $72 .^{42}$ The Western Aphasia Bedside test will be used to assess linguistic skills (information content, fluency, auditory comprehension, repetition, naming and word-finding, reading and writing) and non-linguistic skills (drawing, block design, calculation and praxis) of adults with aphasia. ${ }^{43}$ The Motricity Index is an ordinal scale for limb strength, with six items on each side (three for the arm and three for the leg). The maximum total arm score is $99+1$ (range from 0 to 99), and the same for the leg. ${ }^{44}$ Swallowing assessment will be performed in all patients admitted in the intensive rehabilitation facility with the volume viscosity test (VVT). Patients with abnormal results in the VVT will undergo a videofluoroscopic swallowing study, which is the goldstandard tool to diagnose oropharyngeal dysphagia. The 8-point Penetration Aspiration Scale, Bolus Residue Scale and Functional Oral Intake Scale will be used to define swallow impairment in terms of aspiration/penetration events, residue and oral intake. ${ }^{45-47}$ Anxiety and depression will be assessed with the Hospital Anxiety Depression Scale (HADS), consisting of 14 items, which can be divided into two subscales of seven items each: the anxiety subscale (HADS-A) and the depression subscale (HADSD). The respondent rates each item on a 4-point scale ranging from 0 (absence) to 3 (extreme presence). The total score is out of 42 (21 per subscale). The total HADS score may be regarded as a global measure of physiological distress. ${ }^{48}$

\section{Activity outcomes}

The level of each patient's activity will be assessed with the Barthel Index, which is a widely used standardised scale for assessing functional disability in basic activities of daily living. ${ }^{2627}$ Disability after stroke will be assessed with the
mRS. ${ }^{49}$ Gait speed will be assessed with the $10 \mathrm{~m}$ test: patients will walk a distance of $14 \mathrm{~m}$ ( $2 \mathrm{~m}$ acceleration and deceleration) twice at their maximum speed. The time will be measured and the mean speed calculated $(\mathrm{m} / \mathrm{s}) .{ }^{51}{ }^{52}$ Arm function will be assessed using the Action Research Arm Test, which is divided into four subtests for grasp, grip, pinch and gross arm movement. Performance on each item is rated on a 4-point ordinal scale from 0 to 3 with a maximum score of 57 , a higher score indicating a better level of function. 5354

\section{Participation outcomes}

Health-related quality of life will be assessed using the Stroke Impact Scale-16 which covers eight domains: strength ( 4 items), hand function ( 5 items), mobility (9 items), activities of daily living (10 items), memory (7 items), communication (7 items), emotion (9 items) and handicap. $^{5556}$

\section{Data analysis}

All patient's clinical, demographic and assessment data will be kept in a secure database. Data on recruitment and the transcription of the therapy dose register will also be included. Analyses will be carried out using IBM SPSS Statistics V.24. The continuous variables will be described with the mean and SD or with the median and IQR, depending on their distribution. The categorical variables will be described as percentages. To describe the stroke rehabilitation programme, the amount of time spent on each type of activity during the therapy sessions will be reported as mean and SD. In order to minimise missing data, all questionnaires are user friendly and collected electronically, and all personnel related to the study are trained to identify and engage participants who may be at risk of dropout during follow-up. ANOVA for repeated measures will be used to describe the changes in clinical assessment measures across the four-time points. The last observation carried forward will be used to deal with missing value.

\section{DISCUSSION}

This study will provide a description of an intensive rehabilitation programme for patients with subacute stroke delivered at our inpatient rehabilitation unit. Focusing on the type of activities and the amount of therapy time, the results of this study may bring out new perspectives on how to describe stroke rehabilitation interventions. Another crucial contribution of this study is that the clinical assessments are not limited to the inpatient period, but also extend to the long-term phase. Nowadays, rehabilitation interventions are poorly described in research studies and it is often limited to measure the time spent in each therapy without taking into account the type and amount of activity and tasks performed. ${ }^{5758}$

The amount of rehabilitation therapy contributes to functional recovery after stroke, ${ }^{59}$ but different studies have pointed out a discrepancy between the planned 
therapy hours and the actual practice time. ${ }^{6}{ }^{17-20}$ The optimal dose-response in stroke rehabilitation has not been established and further research is needed to elucidate and better understand the relationship between training intensity and recovery. Lohse et $a t^{60}$ reported a positive relationship between the time scheduled for therapy and therapy outcomes, suggesting that large doses of therapy lead to clinically meaningful improvements, controlling for time after stroke. However, Lang et al have found no evidence of a dose-response effect of task-specific training on functional capacity in people with long-standing upper-limb paresis post stroke. ${ }^{61}$

Therefore, we believe that an agreed description of the rehabilitation programme is the first step required to improve transparency, to ensure fidelity of implementation and to investigate any aspect related to the dose of the stroke rehabilitation programme.

The data collected in this study will rely on the information provided by the therapists, and although all participating therapists have agreed to provide reliable data when reporting therapy sessions, there might be a social desirability bias and inaccuracy. Indeed, we acknowledge that the registry tool is just an estimation of the time spent on specific activities during therapies, which is not comprehensive as far as therapies are concerned. Using more objective tools to measure interventions such as video or auditing records of third parties may not guarantee that the information is more reliable. The therapy activities and time spent in each of them will depend on the individual deficits of each patient. This represents an inherent limitation for the generalisation of results in rehabilitation studies since interventions are tailored to the patient. Another limitation could be the lack of qualitative assessments for the interventions. This study only allows us to quantify the time allocated to each activity during specific treatment sessions, but does not register qualitative or patient-centred aspects. For instance, we will not measure the motivation of the patient, which is an aspect that may influence the effectiveness of the therapies. Moreover, there are other elements of the rehabilitation process that play a crucial role that will not be measured such as the therapeutical relationships established with the patient, the direct and non-direct inputs during therapy sessions, how other staff members (physicians, nurses, nurse assistants, porters) interact with the patient in a supporting manner and environmental factors of our unit favouring recovery. In a similar vein, the therapist's personal abilities to propose the right combination of techniques for each patient and the team workload distribution are aspects that are not controlled in this study. ${ }^{62}$

One of the strengths of this study is that treatment registry data has been agreed between all members of the rehabilitation team, reflecting actual clinical practice. However, future research would be needed in order to check how comprehensive this registry tool is and assess its feasibility. The set of selected clinical outcomes take into account all the dimensions of the ICF and therefore represent a comprehensive view of the consequences of stroke and the effects of rehabilitation.

\section{ETHICS AND DISSEMINATION}

The study will follow the national and international ethical guidelines (Code of Ethics, Declaration of Helsinki) for research in humans and will comply with the legal regulations on data confidentiality (Organic Law 15/1999, of 13 December, on Personal Data Protection). Potential participants will receive oral and written information about the study's objectives and procedures, before deciding whether to provide written informed consent. The BRAIN-CONNECTS study has been evaluated and accepted by the Medical Research Committee at Hospital del Mar Research Institute (Project ID: 34/C/2017). All patients invited to participate in this trial will be asked to agree and sign the written consent in order to participate in the current study.

The findings will be disseminated in clinical seminars, scientific conferences and submitted for publication in peer-reviewed journals.

\section{Author affiliations}

${ }^{1}$ Rehabilitation Research Group, Institut Hospital del Mar d'Investigacions Mediques, Barcelona, Spain

${ }^{2}$ Physiotherapy Degree, Universitat Autonoma de Barcelona, Barcelona, Spain ${ }^{3}$ Cognition and Brain Plasticity Unit, Department of Cognition, Development and Educational Psychology, University of Barcelona and Bellvitge Biomedical Research Institute (IDIBELL), Barcelona, Spain

${ }^{4}$ Escola Universitària d'Infermeria i Teràpia Ocupacional de Terrassa (EUIT), Autonomous University of Barcelona, Terrassa, Spain

${ }^{5}$ Department of Physical Medicine and Rehabilitation, Hospitals del Mar i I'Esperança, Parc de Salut Mar, Barcelona, Spain

${ }^{6}$ Department of Radiology, Biomedical Research Institute Imaging Research Unit, Diagnostic Imaging Institute, Doctor Josep Trueta University Hospital of Girona, Girona, Spain

${ }^{7}$ Centre for Brain Research, The University of Auckland, Auckland, New Zealand

Acknowledgements AGAUR — this research has been partially supported by a grant from 'Secretaria d'Universitats i Recerca del Departament d'Empresa i Coneixement de la Generalitat de Catalunya'. We also would like to thank the multidisciplinary team of therapists of the Hopsital de L'Esperança (Norah Rueda, OT; Ruben Valero, FT and Sonia Nieto, SLP) that have helped in the design of the therapies activity registry. The authors would like to thank all the patients and for participating in this study.

Contributors MMS led the conceptualisation, design and implementation of this research protocol, and was the primary author for this manuscript. AM-P contributed with the implementation, review of this research protocol. ED was a leader in the conceptualisation, design and is a contributing author for this manuscript. JG-S together with ED was a leader in the conceptualisation of the project and also had a role in the design and implementation of the research protocol and was the second author for this manuscript. RB and AG-S participated in the design and implementation of the research protocol. JP helped with funding arrangement. CS contributed to refinement of the research protocol. All authors read and approved the final manuscript.

Funding This Project is funded by Fundació La Marató TV3.

Competing interests None declared.

Patient consent for publication Not applicable.

Provenance and peer review Not commissioned; externally peer reviewed.

Supplemental material This content has been supplied by the author(s). It has not been vetted by BMJ Publishing Group Limited (BMJ) and may not have been peer-reviewed. Any opinions or recommendations discussed are solely those 
of the author(s) and are not endorsed by BMJ. BMJ disclaims all liability and responsibility arising from any reliance placed on the content. Where the content includes any translated material, BMJ does not warrant the accuracy and reliability of the translations (including but not limited to local regulations, clinical guidelines, terminology, drug names and drug dosages), and is not responsible for any error and/or omissions arising from translation and adaptation or otherwise.

Open access This is an open access article distributed in accordance with the Creative Commons Attribution Non Commercial (CC BY-NC 4.0) license, which permits others to distribute, remix, adapt, build upon this work non-commercially, and license their derivative works on different terms, provided the original work is properly cited, appropriate credit is given, any changes made indicated, and the use is non-commercial. See: http://creativecommons.org/licenses/by-nc/4.0/.

\section{ORCID iD}

Monique Messaggi Sartor http://orcid.org/0000-0002-2807-3266

\section{REFERENCES}

1 GBD 2013 DALYs and HALE Collaborators, Murray CJL, Barber $\mathrm{RM}$, et al. Global, regional, and national disability-adjusted life years (DALYs) for 306 diseases and injuries and healthy life expectancy (HALE) for 188 countries, 1990-2013: quantifying the epidemiological transition Lancet 2015;386:2145-91.

2 Hay SI, Abajobir AA, Abate $\mathrm{KH}$, et al. Global, regional, and national disability-adjusted life-years (DALYs) for 333 diseases and injuries and healthy life expectancy (HALE) for 195 countries and territories, 1990-2016: a systematic analysis for the global burden of disease study 2016. Lancet 2017;390:1260-344.

3 Hankey GJ. Stroke. The Lancet 2017;389:641-54.

4 Buntin MB, Colla CH, Deb P, et al. Medicare spending and outcomes after postacute care for stroke and hip fracture. Med Care 2010;48:776-84.

5 Krakauer JW, Carmichael ST, Corbett D, et al. Getting neurorehabilitation right: what can be learned from animal models? Neurorehabil Neural Repair 2012;26:923-31.

6 Winstein CJ, Stein J, Arena R, et al. Guidelines for adult stroke rehabilitation and recovery: a guideline for healthcare professionals from the American heart Association/American stroke association. Stroke 2016;47:e98-169.

7 Miller EL, Murray L, Richards L, et al. Comprehensive overview of nursing and interdisciplinary rehabilitation care of the stroke patient: a scientific statement from the American heart association. Stroke 2010;41:2402-48.

8 Chan L, Sandel ME, Jette AM, et al. Does postacute care site matter? A longitudinal study assessing functional recovery after a stroke. Arch Phys Med Rehabil 2013;94:622-9.

9 Wang $\mathrm{H}$, Camicia M, DiVita M, et al. Early inpatient rehabilitation admission and stroke patient outcomes. Am J Phys Med Rehabil 2015;94:85-100.

10 Deutsch A, Granger CV, Heinemann AW, et al. Poststroke rehabilitation: outcomes and reimbursement of inpatient rehabilitation facilities and subacute rehabilitation programs. Stroke 2006;37:1477-82.

11 Sood N, Huckfeldt PJ, Grabowski DC, et al. The effect of prospective payment on admission and treatment policy: evidence from inpatient rehabilitation facilities. J Health Econ 2013;32:965-79.

12 SAFE. SAFE annual report 2019, 2021. Available: https://www. safestroke.eu/2020/01/05/safe-annual-report-2019-2/

13 Buma F, Kwakkel G, Ramsey N. Understanding upper limb recovery after stroke. Restor Neurol Neurosci 2013;31:707-22.

14 Kwakkel G, Kollen B, Lindeman E. Understanding the pattern of functional recovery after stroke: facts and theories. Restor Neurol Neurosci 2004;22:281-99.

15 Krakauer JW, Carmichael ST, Corbett D, et al. Getting neurorehabilitation right: what can be learned from animal models? Neurorehabil Neural Repair 2012;26:92.

16 Wang H, Camicia M, Terdiman J, et al. Daily treatment time and functional gains of stroke patients during inpatient rehabilitation. Pm $R$ 2013;5:122-8.

17 Zeiler SR, Krakauer JW. The interaction between training and plasticity in the poststroke brain. Curr Opin Neurol 2013;26:609-16.

18 Connell LA, McMahon NE, Simpson LA, et al. Investigating measures of intensity during a structured upper limb exercise program in stroke rehabilitation: an exploratory study. Arch Phys Med Rehabil 2014:95:2410-9.

19 Bernhardt J, Borschmann K, Boyd L, et al. Moving rehabilitation research forward: developing consensus statements for rehabilitation and recovery research. Neurorehabil Neural Repair 2017;31:694-8.
20 Bernhardt J, Hayward KS, Kwakkel G, et al. Agreed definitions and a shared vision for new standards in stroke recovery research: the stroke recovery and rehabilitation roundtable Taskforce. Neurorehabil Neural Repair 2017;31:793-9.

21 DeJong G, Horn SD, Conroy B, et al. Opening the black box of poststroke rehabilitation: stroke rehabilitation patients, processes, and outcomes. Arch Phys Med Rehabil 2005;86:1-7.

22 Lohse KR, Pathania A, Wegman R, et al. On the reporting of experimental and control therapies in stroke rehabilitation trials: a systematic review. Arch Phys Med Rehabil 2018;99:1424-32.

23 Ballinger C, Ashburn A, Low J, et al. Unpacking the black box of therapy - a pilot study to describe occupational therapy and physiotherapy interventions for people with stroke. Clin Rehabil 1999;13:301-9.

24 van Vliet PM, Lincoln NB, Robinson E. Comparison of the content of two physiotherapy approaches for stroke. Clin Rehabil 2001;15:398-414.

25 Bode RK, Heinemann AW, Semik P, et al. Patterns of therapy activities across length of stay and impairment levels: peering inside the "black box" of inpatient stroke rehabilitation. Arch Phys Med Rehabil 2004;85:1901-8.

26 Lang CE, MacDonald JR, Gnip C. Counting repetitions: an observational study of outpatient therapy for people with hemiparesis post-stroke. J Neurol Phys Ther 2007;31:3-10.

27 Tyson SF, Selley A. The development of the stroke physiotherapy intervention recording tool (spirit). Disabil Rehabil 2004;26:1184-8.

28 Veerbeek JM, van Wegen E, van Peppen R, et al. What is the evidence for physical therapy poststroke? A systematic review and meta-analysis. PLoS One 2014;9:e87987.

29 Jette AM. The post-stroke rehabilitation outcomes project. Arch Phys Med Rehabil 2005;86:124-5.

30 Stummer C, Verheyden G, Putman K, et al. Predicting sickness impact profile at six months after stroke: further results from the European multi-center CERISE study. Disabil Rehabil 2015;37:942-50.

31 Graham JE, Granger CV, Karmarkar AM, et al. The uniform data system for medical rehabilitation: report of follow-up information on patients discharged from inpatient rehabilitation programs in 20022010. Am J Phys Med Rehabil 2014;93:231-44.

32 von Elm E, Altman DG, Egger M, et al. The strengthening the reporting of observational studies in epidemiology (STROBE) statement: guidelines for reporting observational studies. J Clin Epidemiol 2008;61:344-9.

33 Vivanco-Hidalgo RM, Abilleira S, Salvat-Plana M, et al. Innovation in systems of care in acute phase of ischemic stroke. The experience of the Catalan stroke programme. Front Neurol 2018;9:427.

34 Marrugat J, Vila J, Pavesi M, et al. [Estimation of the sample size in clinical and epidemiological investigations]. Med Clin 1998;111:267-76.

35 Langhorne P, Bernhardt J, Kwakkel G. Stroke rehabilitation. The Lancet 2011;377:1693-702.

36 Aguirrezabal A, Duarte E, Rueda N, et al. Effects of information and training provision in satisfaction of patients and carers in stroke rehabilitation. NeuroRehabilitation 2013;33:639-47.

37 Mahoney FI, Barthel DW. Functional evaluation: the BARTHEL index. Md State Med J 1965;14:61-5.

38 Cid-Ruzafa J, Damián-Moreno J. Evaluating physical incapacity: the Barthel index. Rev Esp Salud Pública 1997;71:127-37.

39 Holden MK, Gill KM, Magliozzi MR. Gait assessment for neurologically impaired patients. standards for outcome assessment. Phys Ther 1986;66:1530-9.

40 Page SJ, Fulk GD, Boyne P. Clinically important differences for the upper-extremity Fugl-Meyer scale in people with minimal to moderate impairment due to chronic stroke. Phys Ther 2012:92:791-8.

41 Nasreddine ZS, Phillips NA, Bédirian V, et al. The Montreal cognitive assessment, MoCA: a brief screening tool for mild cognitive impairment. J Am Geriatr Soc 2005;53:695-9.

42 Marin RS, Biedrzycki RC, Firinciogullari S. Reliability and validity of the apathy evaluation scale. Psychiatry Res 1991;38:143-62.

43 Risser AH, Spreen O. The Western aphasia battery. J Clin Exp Neuropsychol 1985;7:463-70.

44 Collin C, Wade D. Assessing motor impairment after stroke: a pilot reliability study. J Neurol Neurosurg Psychiatry 1990;53:576-9.

45 Logemann JA. Measurement of swallow from videofluorographic studies. Austin: Pro-ed, 1993.

46 Rommel N, Borgers C, Van Beckevoort D, et al. Bolus residue scale: an easy-to-use and reliable videofluoroscopic analysis tool to score bolus residue in patients with dysphagia. Int J Otolaryngol $2015 ; 2015: 1-7$ 
47 Crary MA, Mann GDC, Groher ME. Initial psychometric assessment of a functional oral intake scale for dysphagia in stroke patients. Arch Phys Med Rehabil 2005;86:1516-20.

48 Aben I, Verhey F, Lousberg R, et al. Validity of the Beck depression inventory, hospital anxiety and depression scale, SCL-90, and Hamilton depression rating scale as screening instruments for depression in stroke patients. Psychosomatics 2002;43:386-93.

49 van Swieten JC, Koudstaal PJ, Visser MC, et al. Interobserver agreement for the assessment of handicap in stroke patients. Stroke 1988;19:604-7.

50 Bonita R, Beaglehole R. Recovery of motor function after stroke. Stroke 1988;19:1497-500.

51 Pohl M, Werner C, Holzgraefe M, et al. Repetitive locomotor training and physiotherapy improve walking and basic activities of daily living after stroke: a single-blind, randomized multicentre trial (Deutsche gang trainer studie, DEGAS). Clin Rehabil 2007;21:17-27.

52 Perera S, Mody SH, Woodman RC, et al. Meaningful change and responsiveness in common physical performance measures in older adults. J Am Geriatr Soc 2006;54:743-9.

53 Hsieh CL, Hsueh IP, Chiang FM, et al. Inter-Rater reliability and validity of the action research arm test in stroke patients. Age Ageing 1998;27:107-13.

54 Lang CE, Wagner JM, Dromerick AW, et al. Measurement of upperextremity function early after stroke: properties of the action research arm test. Arch Phys Med Rehabil 2006;87:1605-10.
55 Richardson M, Campbell N, Allen L, et al. The stroke impact scale: performance as a quality of life measure in a community-based stroke rehabilitation setting. Disabil Rehabil 2016;38:1425-30

56 Duncan PW, Wallace D, Lai SM, et al. The stroke impact scale version 2.0. Evaluation of reliability, validity, and sensitivity to change. Stroke 1999;30:2131-40.

57 Glasziou P, Meats E, Heneghan C, et al. What is missing from descriptions of treatment in trials and reviews? BMJ 2008;336:1472-4.

58 Hoffmann TC, Glasziou PP, Boutron I, et al. Better reporting of interventions: template for intervention description and replication (TIDieR) checklist and guide. BMJ 2014;348:g1687.

59 Foley N, McClure JA, Meyer M, et al. Inpatient rehabilitation following stroke: amount of therapy received and associations with functional recovery. Disabil Rehabil 2012;34:2132-8.

60 Lohse KR, Lang CE, Boyd LA. Is more better? using metadata to explore dose-response relationships in stroke rehabilitation. Stroke 2014;45:2053-8.

61 Lang CE, Strube MJ, Bland MD, et al. Dose response of task-specific upper limb training in people at least 6 months poststroke: a phase II, single-blind, randomized, controlled trial. Ann Neurol 2016;80:342-54.

62 Negrini S. Evidence in rehabilitation medicine: between facts and Prejudices. Am J Phys Med Rehabil 2019;98:88-96. 